Max-Planck-Institut für demografische Forschung

Max Planck Institute for Demographic Research

Konrad-Zuse-Strasse 1 - D-18057 Rostock · GERMANY

Tel +49 (0) 3812081 - 0; Fax +49 (0) 3812081 - 202;

http://www.demogr.mpg.de

MPIDR WORKING PAPER WP 2010-016

FEBRUARY 2010

\title{
The ages of extremal impact on life disparity caused by averting deaths
}

Peter Wagner (wagner@demogr.mpg.de)

This working paper has been approved for release by: James W. Vaupel (jwv@demogr.mpg.de), Head of the Laboratory of Survival and Longevity.

(C) Copyright is held by the authors.

Working papers of the Max Planck Institute for Demographic Research receive only limited review. Views or opinions expressed in working papers are attributable to the authors and do not necessarily reflect those of the Institute. 


\title{
The ages of extremal impact on life disparity caused by averting deaths
}

\author{
Peter Wagner \\ January 10, 2010 \\ Max Planck Institute for Demographic Research \\ Konrad-Zuse-Straße 1 \\ 18057 Rostock, Germany \\ e-mail: wagner@demogr.mpg.de
}

\begin{abstract}
This paper is concerned with sensitivity analysis of life disparity with respect to changes in mortality rates. Recently Zhang and Vaupel introduced a "threshold age", such that averting deaths before that age reduces disparity, while averting deaths after that age increases disparity. We provide a refinement to this result by characterizing the ages at which averting deaths has an extremal impact on life disparity. A procedure is given for approaching the threshold age numerically. The results are illustrated using data for the female populations of Denmark, the US, Japan and France in 2005.
\end{abstract}

\section{Contents}

1. Introduction 2

2. Main results 4

2.1. The effect on life disparity caused by averting deaths . . . . . . 4

2.2. Approaching the threshold age numerically . . . . . . . . . 5 5

$\begin{array}{lr}3 . & \text { Illustrations }\end{array}$

3.1. Constant hazards . . . . . . . . . . . . . . . . . 6

3.2. Numerical findings . . . . . . . . . . . . . . . . . 7

4. Proofs 10

4.1. Proof of Theorem 2.1 . . . . . . . . . . . . . . 10

4.2. Proof of Theorem 2.2 . . . . . . . . . . . . . . 13

5. Appendix: Calculating the perturbed life disparity 14

$\begin{array}{ll}\text { References } & 15\end{array}$ 


\section{Introduction}

Following Keyfitz's idea that everybody dies prematurely since every death deprives the person involved of the remainder of his expectation of life [Key77, p.61-68], the measure $e^{\dagger}$ for the average life expectancy lost due to death, has widely been studied in the literature. It first appeared in [Mit78] and was developed further by Vaupel [Vau86] and recently in [VCR03], [ZV08] and $\left[\mathrm{SAZ}^{+} 09\right]$. Zhang and Vaupel [ZV09] initiated a new direction of analysis, by studying the impact on $e^{\dagger}$ of a concentrated decrease in mortality at age $a$.

Life disparity is measured by life expectancy lost due to death

$$
e^{\dagger}=\int_{0}^{\infty} e(a) d(a) d a
$$

where $e(a)$ is the remaining life expectancy at age $a$

$$
e(a)=\frac{1}{l(a)} \int_{a}^{\infty} l(x) d x
$$

$d(a)$ is the life table distribution of deaths

$$
d(a)=l(a) \mu(a)
$$

$l(a)$ is the probability of survival to age $a$

$$
l(a)=\exp \left(-\int_{0}^{a} \mu(x) d x\right)
$$

and $\mu(a)$ is the age-specific hazard of death. Note that, by equations (1.4) and $(1.3)$,

$$
\frac{d}{d a}[1-l(a)]=l(a) \mu(a)=d(a)
$$

Letting $H(a)$ be the cumulative hazard function

$$
H(a)=\int_{0}^{a} \mu(x) d x
$$

in [ZV09], a perturbation is considered that alters $H(a)$ by the step function with a single negative step of size $s$ at age $a$ to get the new cumulative hazard function

$$
H_{a, s}(x)=H(x)-s \cdot \mathbb{1}_{[a, \infty)}(x)
$$

It can be derived, as done in the Appendix, that the corresponding new value of life disparity satisfies

$$
e_{a, s}^{\dagger}=e^{\dagger}+[\exp (s)-1] l(a) e(a)\left[H(a)-1+\frac{e^{\dagger}(a)}{e(a)}\right]
$$


where $e^{\dagger}(a)$ is life expectancy lost due to death among people surviving to age $a$

$$
e^{\dagger}(a)=\frac{1}{l(a)} \int_{a}^{\infty} e(x) d(x) d x
$$

Now

$$
\frac{\partial}{\partial s} e_{a, s}^{\dagger}=\exp (s) l(a) e(a)\left[H(a)-1+\frac{e^{\dagger}(a)}{e(a)}\right]
$$

and

$$
\left.\frac{\partial}{\partial s} e_{a, s}^{\dagger}\right|_{s=0}=l(a) e(a)\left[H(a)-1+\frac{e^{\dagger}(a)}{e(a)}\right]
$$

In [ZV09], the function $k$, where

$$
\begin{aligned}
k(a) & =\left.\frac{1}{l(a)} \frac{\partial}{\partial s} e_{a, s}^{\dagger}\right|_{s=0} \\
& =e(a)\left[H(a)-1+\frac{e^{\dagger}(a)}{e(a)}\right] \\
& =e^{\dagger}(a)+e(a)[H(a)-1]
\end{aligned}
$$

is analysed and shown to have a positive first derivative at every root, from which it follows that $k$ has at most one root. It is shown that $k(0)<0$ implies the existence of a unique root $a^{\dagger}$ (then, of course, positive), $k(a)<0$ for $a<a^{\dagger}$ and $k(a)>0$ for $a>a^{\dagger}$, and that $k(0) \geq 0$ implies $k(a)>0$ for $a>0$.

Since, by equation (1.10), $k(a)$ and $\left.\frac{\partial}{\partial s} e_{a, s}^{\dagger}\right|_{s=0}$ have the same sign, and since $k(0)=e^{\dagger}-e(0)$, this is equivalent to saying

Theorem 1.1 There are the following three cases:

(i) $\frac{e^{\dagger}}{e(0)}<1 \Rightarrow \exists a^{\dagger}>0$ :

$$
\begin{aligned}
& \forall a<\left.a^{\dagger} \Rightarrow \frac{\partial}{\partial s} e_{a, s}^{\dagger}\right|_{s=0}<0 \\
& a=\left.a^{\dagger} \Rightarrow \frac{\partial}{\partial s} e_{a, s}^{\dagger}\right|_{s=0}=0 \\
& \forall a>\left.a^{\dagger} \Rightarrow \frac{\partial}{\partial s} e_{a, s}^{\dagger}\right|_{s=0}>0
\end{aligned}
$$

(ii) $\frac{e^{\dagger}}{e(0)}=1 \Rightarrow a^{\dagger}=0$ :

$$
\begin{aligned}
& a=\left.a^{\dagger} \Rightarrow \frac{\partial}{\partial s} e_{a, s}^{\dagger}\right|_{s=0}=0 \\
& \forall a>\left.a^{\dagger} \Rightarrow \frac{\partial}{\partial s} e_{a, s}^{\dagger}\right|_{s=0}>0
\end{aligned}
$$

(iii) $\frac{e^{\dagger}}{e(0)}>1$ :

$$
\left.\forall a \Rightarrow \frac{\partial}{\partial s} e_{a, s}^{\dagger}\right|_{s=0}>0
$$


The unique root $a^{\dagger}$ of $k(a)$ and $\left.\frac{\partial}{\partial s} e_{a, s}^{\dagger}\right|_{s=0}$, if it exists, that is, if $\frac{e^{\dagger}}{e(0)} \leq 1$, is called threshold age.

In this paper we provide a refinement to the above result by characterizing the ages at which averting deaths has an extremal impact (or, in other words, effect) on life disparity. In addition, a procedure is given for approaching the threshold age numerically. The results are illustrated using data for the female populations of Denmark, the US, France and Japan in 2005.

The paper is organised as follows. The main results are presented in Section 2. An explicit example as well as some numerical results using data from the Human Mortality Database are given in Section 3. The proofs of the theorems are collected in Section 4. A formula for the perturbed life disparity is derived in the Appendix.

\section{Main results}

The function $\left.\frac{\partial}{\partial s} e_{a, s}^{\dagger}\right|_{s=0}$ (cf. (1.9)) measures the effect on life disparity of averting deaths at age $a$. It turns out that looking at this function directly allows us to make interesting statements about its behaviour, beyond those about the sign obtained by looking at $k$ (cf. (1.10)) and summarised in Theorem 1.1.

\subsection{The effect on life disparity caused by averting deaths}

The main result of this paper is the following

Theorem 2.1 The function $\varphi$, defined by

$$
\varphi(a)=\left.\frac{\partial}{\partial s} e_{a, s}^{\dagger}\right|_{s=0}
$$

has the following properties.

(i) Let $\tilde{a}$, the age of cumulative hazard unity, be defined via

$$
H(\tilde{a})=1
$$

Then $\varphi$ is strictly increasing on $[0, \tilde{a}]$ and strictly decreasing and strictly positive on $[\tilde{a}, \infty)$, having

- a global maximum of $\varphi(\tilde{a})=l(\tilde{a}) e^{\dagger}(\tilde{a})$ at $a=\tilde{a}$ and

- a local minimum of $\varphi(0)=e^{\dagger}(0)-e(0)$ at $a=0$.

More precisely,

$$
\frac{d}{d a} \varphi(a)=l(a)[1-H(a)]
$$


(ii) If, for some $\varepsilon>0$ and $A \geq 0$, we have

$$
\mu(a) \geq \varepsilon \quad \forall a \geq A
$$

then

$$
\lim _{a \rightarrow \infty} \varphi(a)=0
$$

(iii) Let $a^{*}$ be defined by

$$
H\left(a^{*}\right)=2
$$

Then $\varphi$ is strictly concave on $\left[0, a^{*}\right]$ and strictly convex on $\left[a^{*}, \infty\right)$. More precisely,

$$
\frac{d^{2}}{d a^{2}} \varphi(a)=d(a)[H(a)-2]
$$

Regarding the existence of $\tilde{a}$ and $a^{*}$, note that, by equation (1.6), $H$ is strictly increasing with $H(0)=0$, and that $\lim _{a \rightarrow \infty} H(a)=\infty$ is generally assumed.

Theorem 2.1(i) implies that $\varphi$ has at most one root, which exists if and only if $\varphi(0) \leq 0 \Longleftrightarrow e^{\dagger}(0)-e(0) \leq 0 \Longleftrightarrow \frac{e^{\dagger}}{e(0)} \leq 1$, so that Theorem 2.1(i) implies Theorem 1.1. Refining Theorem 1.1, our Theorem 2.1 highlights some helpful monotonicity properties and draws attention to $\tilde{a}$ and 0 , the ages of extremal effect on life disparity caused by averting deaths.

Consider ages $a>a^{\dagger}$, if $a^{\dagger}$ exists, and consider any age otherwise. By Theorem 1.1, life disparity increases by averting deaths at any such age. More precisely, by Theorem 2.1(i), the increase in life disparity by averting deaths is the higher the closer the age is to $\tilde{a}$, where the increase is highest.

Consider ages $a<a^{\dagger}$, if $a^{\dagger}$ exists. By Theorem 1.1, life disparity decreases by averting deaths at any such age. More precisely, by Theorem 2.1(i), the decrease in life disparity by averting deaths is the higher the closer the age is to 0 , where the decrease is highest.

\subsection{Approaching the threshold age numerically}

According to Theorem 2.1(iii), since $\tilde{a}<a^{*}$ clearly holds, $\varphi$ is strictly concave on $[0, \tilde{a}]$. This is the property we can make use of for approximating $a^{\dagger}$, provided such an approximation makes sense, that is, $\varphi(0)<0$.

First, we can construct a strictly increasing sequence that approaches $a^{\dagger}$ from below. Second, we can construct a strictly decreasing sequence that approaches $a^{\dagger}$ from above.

Theorem 2.2 Assume $\frac{e^{\dagger}}{e(0)}<1$ and let $a^{\dagger}$ be the threshold age, defined by $\varphi\left(a^{\dagger}\right)=0$. 
(i) The sequence $\left(x_{n}\right)$, defined by

$$
x_{n+1}=x_{n}+e\left(x_{n}\right)-\frac{e^{\dagger}\left(x_{n}\right)}{1-H\left(x_{n}\right)}, \quad x_{0}=0
$$

strictly increases and rapidly (quadratically) converges to $a^{\dagger}$. In particular,

$$
x_{1}=e(0)-e^{\dagger}(0)
$$

is a first non-trivial approximation of $a^{\dagger}$ from below.

(ii) The sequence $\left(y_{n}\right)$, defined by

$$
y_{n+1}=y_{n} \cdot \frac{e(0)-e^{\dagger}(0)}{l\left(y_{n}\right) e\left(y_{n}\right)\left[H\left(y_{n}\right)-1\right]+l\left(y_{n}\right) e^{\dagger}\left(y_{n}\right)+e(0)-e^{\dagger}(0)}, \quad y_{0}=\tilde{a}
$$

strictly decreases and (linearly) converges to $a^{\dagger}$. In particular,

$$
y_{1}=\tilde{a} \cdot \frac{e(0)-e^{\dagger}(0)}{l(\tilde{a}) e^{\dagger}(\tilde{a})+e(0)-e^{\dagger}(0)}
$$

is a first non-trivial approximation of $a^{\dagger}$ from above.

Concluding for $x_{1}$ and $y_{1}$ of Theorem 2.2, we have $a^{\dagger} \in\left(x_{1}, y_{1}\right) \subset[0, \tilde{a}]$. This, of course, does not locate $a^{\dagger}$ precisely, but constitutes new non-trivial bounds on $a^{\dagger}$. First, we can say that life disparity decreases by averting deaths at any age $a \leq x_{1}=e(0)-e^{\dagger}(0)$ (with the decrease being the higher the closer the age is to 0 ). Second, we can say that life disparity increases by averting deaths at any age $a \geq y_{1}=\tilde{a} \cdot \frac{e(0)-e^{\dagger}(0)}{l(\tilde{a}) e^{\dagger}(\tilde{a})+e(0)-e^{\dagger}(0)}$ (with the increase being the higher the closer the age is to $\tilde{a}$ ).

\section{Illustrations}

\subsection{Constant hazards}

Constant hazards are unrealistic for human populations, where, roughly speaking, between ages 30 and 90 the hazard is exponential. However, hypothetical populations with constant hazards of death can be used to illustrate Theorem 2.1. The effect on life disparity caused by averting deaths at age $a$ should be strictly increasing from age 0 to $\tilde{a}$ and strictly decreasing (and strictly positive) from age $\tilde{a}$ onwards.

Indeed, suppose

$$
\mu(a)=C
$$

By equation (1.6),

$$
H(a)=\int_{0}^{a} \mu(x) d x=C a
$$


By equations (1.4) and (1.6),

$$
l(a)=\exp (-C a)
$$

By equation (1.3),

$$
d(a)=l(a) \mu(a)=C \exp (-C a)
$$

By equation (1.2),

$$
\begin{aligned}
e(a) & =\frac{1}{l(a)} \int_{a}^{\infty} l(x) d x \\
& =\exp (C a) \int_{a}^{\infty} \exp (-C x) d x \\
& =\exp (C a)\left[\frac{-1}{C} \exp (-C x)\right]_{a}^{\infty} \\
& =\exp (C a)\left[\frac{1}{C} \exp (-C a)\right]^{\infty}=\frac{1}{C}
\end{aligned}
$$

By equations (1.8) and (1.5),

$$
\begin{aligned}
e^{\dagger}(a) & =\frac{1}{l(a)} \int_{a}^{\infty} e(x) d(x) d x \\
& =\frac{1}{C l(a)}[1-l(x)]_{a}^{\infty}=\frac{1}{C}
\end{aligned}
$$

Finally, by equations (4.2), (4.1), (3.2), (3.3), (3.1) and (3.4),

$$
\begin{aligned}
\varphi(a) & =l(a) e(a) \psi(a) \\
& =l(a) e(a)\left[H(a)-1+\frac{e^{\dagger}(a)}{e(a)}\right] \\
& =\exp (-C a) \frac{1}{C}\left[C a-1+\frac{\frac{1}{C}}{\frac{1}{C}}\right] \\
& =a \exp (-C a)
\end{aligned}
$$

Clearly, $a^{\dagger}=0$. Since $\tilde{a}$ is defined by $1=H(\tilde{a})=C \tilde{a}$, we have $\tilde{a}=\frac{1}{C}$. It is straightforward to see that $\varphi$ is indeed strictly increasing from age 0 to $\tilde{a}$ and strictly decreasing (and strictly positive) from age $\tilde{a}$ onwards.

\subsection{Numerical findings}

To illustrate the theoretical results, we have computed several relevant quantities for four life tables from the Human Mortality Database 2010 [Dat10]. In the figures below the function $\varphi$, representing the effect on life disparity caused by averting deaths (see Theorem 2.1), is displayed by a solid line. The approximations $x_{1}$ and $y_{1}$ of the threshold age (see Theorem 2.2) are obtained by the intersections of the dashed lines and the age axis. 


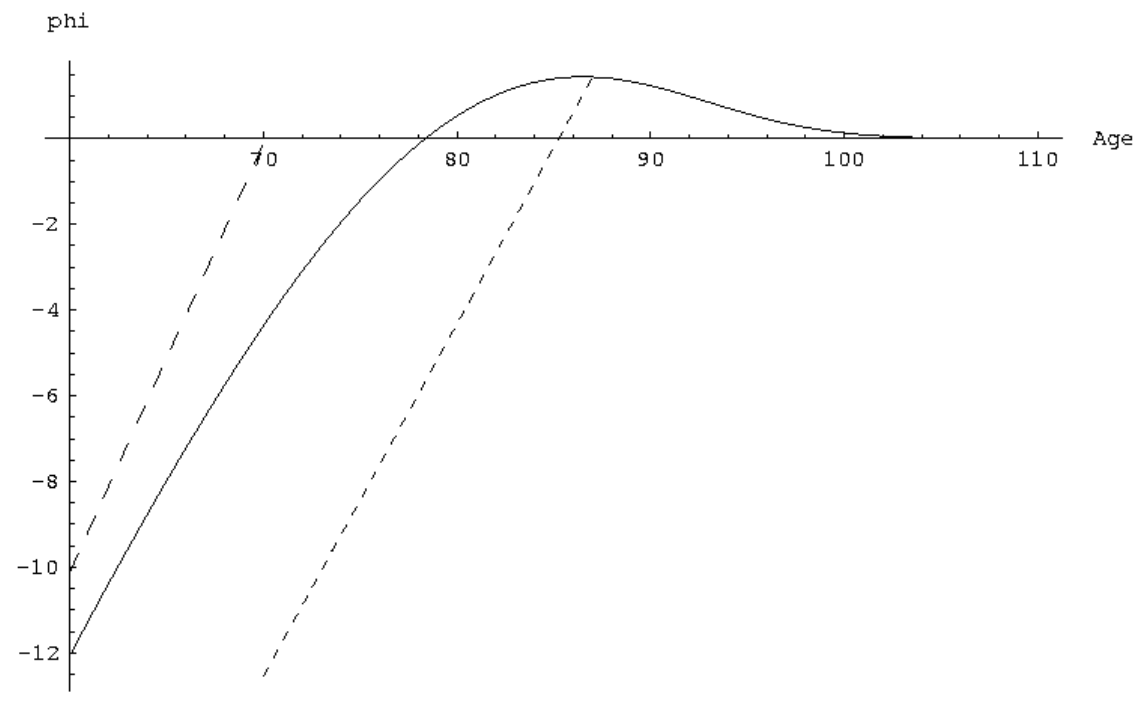

Figure 1: The effect on life disparity caused by averting deaths for Danish females in 2005

For females in Denmark in 2005, the age of cumulative hazard unity $\tilde{a}$ is about 87 years, and $x_{1}, a^{\dagger}$ and $y_{1}$ are approximately 70,78 and 85 years, respectively, as shown in Figure 1. For US-females in 2005, $\tilde{a}$ is around 87 years, and $x_{1}, a^{\dagger}$ and $y_{1}$ are approximately 69,79 and 85 years, respectively, as shown in Figure 2. For females in France in 2005, $\tilde{a}$ is about 90 years, and $x_{1}, a^{\dagger}$ and $y_{1}$ are approximately 74,83 and 88 years, respectively, as shown in Figure 3. Finally, for Japanese females in 2005, $\tilde{a}$ is around 91 years, and $x_{1}, a^{\dagger}$ and $y_{1}$ are approximately 76,84 and 89 years, respectively, as shown in Figure 4. Note that the four obtained threshold ages concur with Figure 2 of [ZV09]. Note further that our Figure 2 concurs with Figure 1 of [ZV09].

It is evident that in all four cases the approximations $x_{1}$ and $y_{1}$ of $a^{\dagger}$ lie within ten years of $a^{\dagger}$, and the arithmetic mean of $x_{1}$ and $y_{1}$ lies within two years of $a^{\dagger}$. In fact, $\tilde{a}$ also lies within ten years of $a^{\dagger}$, and the arithmetic mean of $x_{1}$ and $\tilde{a}$ even lies within one year of $a^{\dagger}$. Whether the arithmetic mean of $x_{1}$ and $\tilde{a}$ is, in general, a better approximation of $a^{\dagger}$ than the arithmetic mean of $x_{1}$ and $y_{1}$, would be an interesting question for future research. If so, it would emphasise the importance of $\tilde{a}$, the age of cumulative hazard unity, and, due to the simple definitions of $x_{1}$ and $\tilde{a}$, improve our understanding of $a^{\dagger}$.

It should be mentioned that in all four cases, the extremal effects on life disparity caused by averting deaths are far from being equal. Indeed, for Denmark, $\varphi(0)=-x_{1} \approx-70.12$ and $\varphi(87) \approx 1.44$ with $-\varphi(0) / \varphi(87) \approx 48.7$, for the US, $\varphi(0)=-x_{1} \approx-68.87$ and $\varphi(87) \approx 1.56$ with $-\varphi(0) / \varphi(87) \approx 44.1$, for France, $\varphi(0)=-x_{1} \approx-74.08$ and $\varphi(90) \approx 1.29$ with $-\varphi(0) / \varphi(90) \approx 57.4$, and 


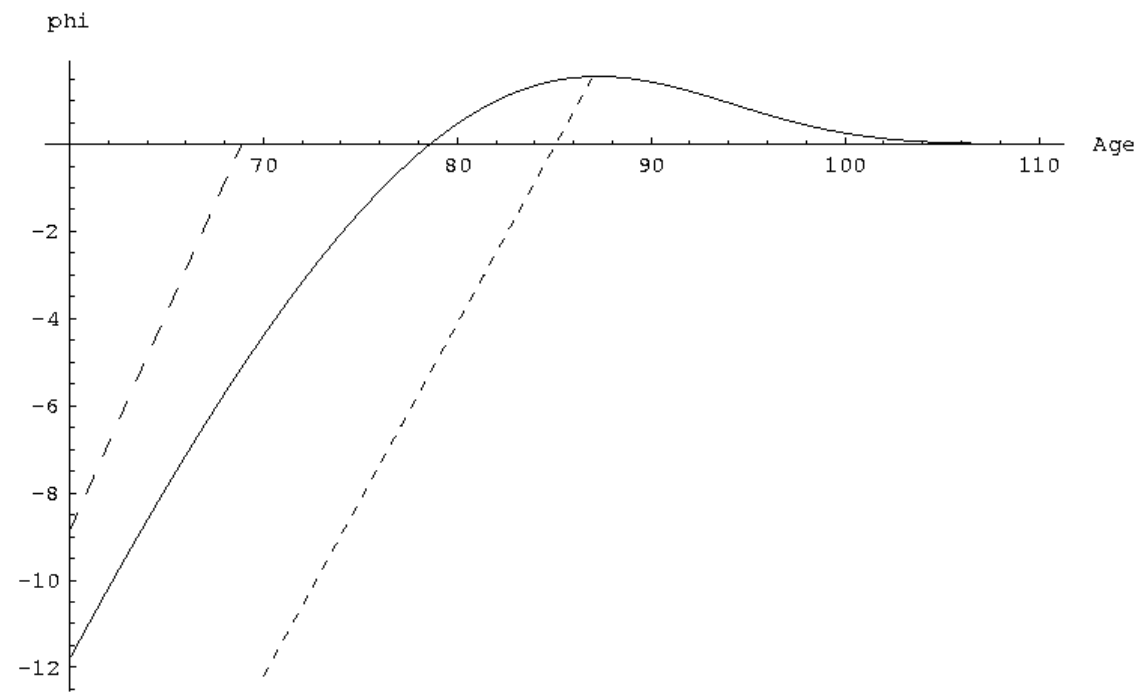

Figure 2: The effect on life disparity caused by averting deaths for US-females in 2005

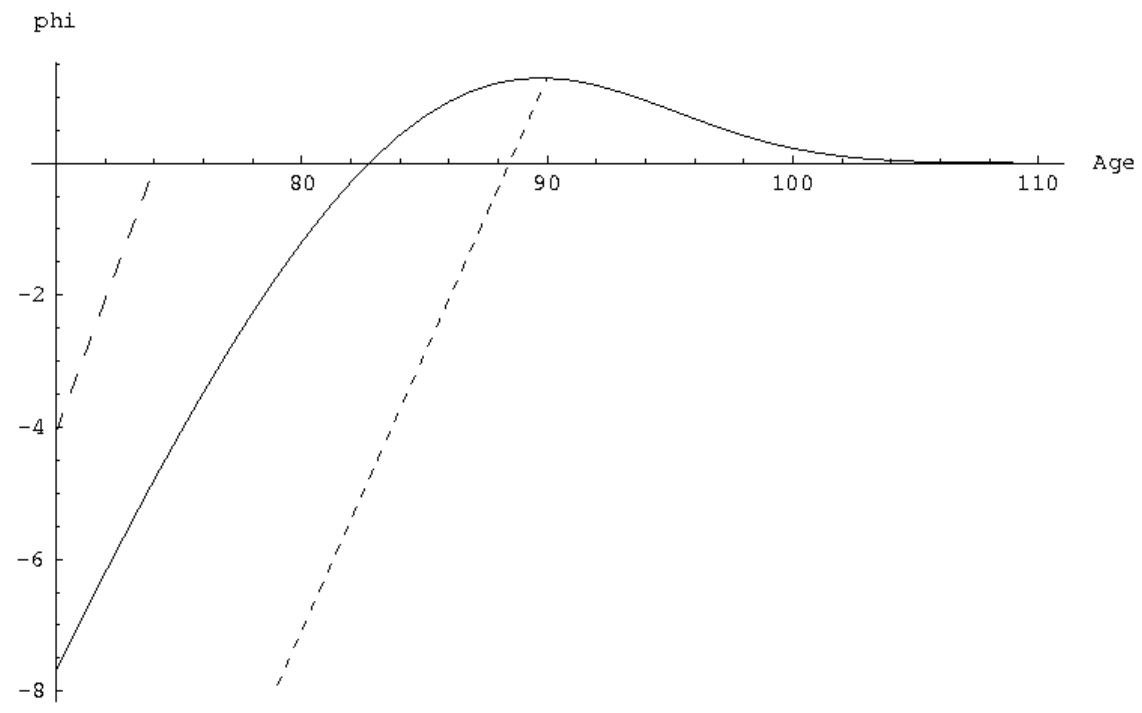

Figure 3: The effect on life disparity caused by averting deaths for French females in 2005 
for Japan, $\varphi(0)=-x_{1} \approx-75.88$ and $\varphi(91) \approx 1.34$ with $-\varphi(0) / \varphi(91) \approx 56.6$, so that, in absolute value, the effect on life disparity by averting deaths at age zero is roughly 50 times larger than the effect on life disparity by averting deaths at age $\tilde{a}$. Studying this ratio for other life tables and possible implications might be another interesting direction of future research.

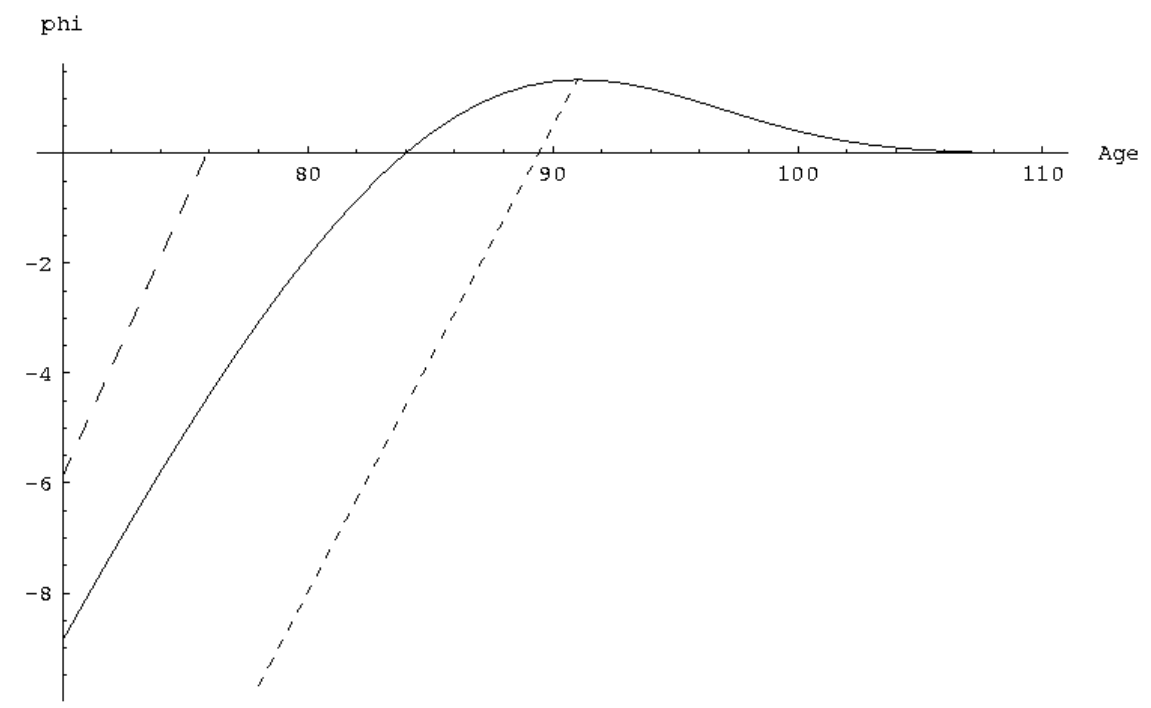

Figure 4: The effect on life disparity caused by averting deaths for Japanese females in 2005

\section{Proofs}

\subsection{Proof of Theorem 2.1}

For proving Theorem 2.1, we need the following result.

Lemma 4.1 The function $\psi$, defined by

$$
\psi(a)=H(a)-1+\frac{e^{\dagger}(a)}{e(a)}
$$

is strictly increasing. More precisely,

$$
\frac{d}{d a} \psi(a)=\frac{e^{\dagger}(a)}{e(a)^{2}}>0
$$


Proof. By equations (1.6), (1.2), (1.8) and (1.3),

$$
\begin{aligned}
\frac{d}{d a} \psi(a) & =\frac{d}{d a} H(a)+\frac{d}{d a} \frac{e^{\dagger}(a)}{e(a)} \\
& =\frac{d}{d a} \int_{0}^{a} \mu(x) d x+\frac{d}{d a} \frac{l(a) e^{\dagger}(a)}{l(a) e(a)} \\
& =\mu(a)+\frac{\left[\frac{d}{d a} \int_{a}^{\infty} e(x) d(x) d x\right] l(a) e(a)-l(a) e^{\dagger}(a)\left[\frac{d}{d a} \int_{a}^{\infty} l(x) d x\right]}{[l(a) e(a)]^{2}} \\
& =\mu(a)+\frac{[-e(a) d(a)] l(a) e(a)-l(a) e^{\dagger}(a)[-l(a)]}{[l(a) e(a)]^{2}} \\
& =\mu(a)-\frac{d(a)}{l(a)}+\frac{e^{\dagger}(a)}{e(a)^{2}} \\
& =\frac{e^{\dagger}(a)}{e(a)^{2}}>0
\end{aligned}
$$

showing that $\psi$ is a strictly increasing function.

\section{Proof of part (i)}

By equations (1.9), (2.1) and (4.1),

$$
\varphi(a)=l(a) e(a) \psi(a)
$$

First, by Lemma 4.1 and equation (4.1), we have $\psi(a)>\psi(\tilde{a})=\frac{e^{\dagger}(\tilde{a})}{e(\tilde{a})}>0$, for all $a>\tilde{a}$. Consequently, by equations (4.2) and (1.2), $\varphi$ is strictly positive on $[\tilde{a}, \infty)$.

Then, by equations (4.2), (1.2), (4.1) and Lemma 4.1, we have

$$
\begin{aligned}
\frac{d}{d a} \varphi(a) & =\frac{d}{d a}[l(a) e(a) \psi(a)] \\
& =\left\{\frac{d}{d a}[l(a) e(a)]\right\} \psi(a)+[l(a) e(a)] \frac{d}{d a} \psi(a) \\
& =\left[\frac{d}{d a} \int_{a}^{\infty} l(x) d x\right]\left[H(a)-1+\frac{e^{\dagger}(a)}{e(a)}\right]+[l(a) e(a)] \frac{e^{\dagger}(a)}{e(a)^{2}} \\
& =[-l(a)]\left[H(a)-1+\frac{e^{\dagger}(a)}{e(a)}\right]+l(a) \frac{e^{\dagger}(a)}{e(a)} \\
& =l(a)[1-H(a)]
\end{aligned}
$$

So the first derivative of $\varphi$ is strictly positive on $[0, \tilde{a})$ and strictly negative on $(\tilde{a}, \infty)$. Thus, $\varphi$ is strictly increasing on $[0, \tilde{a}]$ and strictly decreasing on $[\tilde{a}, \infty)$. Consequently, $\varphi$ has a global maximum at $a=\tilde{a}$, where its value, by equations (4.2) and (4.1), is

$$
\varphi(\tilde{a})=l(\tilde{a}) e^{\dagger}(\tilde{a})
$$

and a local minimum at $a=0$, where its value, by equations (4.2) and (4.1), is

$$
\varphi(0)=e^{\dagger}(0)-e(0)
$$




\section{Proof of part (ii)}

By equations (4.2), (4.1), (1.2), (1.8), (1.4) and (1.6),

$$
\begin{aligned}
\varphi(a) & =l(a) e(a) \psi(a) \\
& =l(a) e(a)\left[H(a)-1+\frac{e^{\dagger}(a)}{e(a)}\right] \\
& =H(a) \int_{a}^{\infty} l(x) d x-\int_{a}^{\infty} l(x) d x+\int_{a}^{\infty} e(x) d(x) d x \\
& =H(a) \int_{a}^{\infty} \exp [-H(x)] d x-\int_{a}^{\infty} l(x) d x+\int_{a}^{\infty} e(x) d(x) d x
\end{aligned}
$$

Clearly, $\lim _{a \rightarrow \infty} \int_{a}^{\infty} l(x) d x=0$ and $\lim _{a \rightarrow \infty} \int_{a}^{\infty} e(x) d(x) d x=0$, so that it remains to show $\lim _{a \rightarrow \infty} H(a) \int_{a}^{\infty} \exp [-H(x)] d x=0$.

Let $c=\frac{\varepsilon}{2}$. Since $\mu(a) \geq \varepsilon$ for all $a \geq A$, we have

$$
[H(x)-c x]-[H(a)-c a]=\int_{a}^{x}[\mu(y)-c] d y \geq 0 \quad \forall x \geq a \geq A
$$

and

$$
H(a)-c a \geq \int_{A}^{a} \mu(x) d x-c a \geq c a-2 c A \quad \forall a \geq A
$$

It follows from equation (4.5) that

$$
\begin{aligned}
\int_{a}^{\infty} \exp [-H(x)] d x & =\int_{a}^{\infty} \exp \{-[H(x)-c x]-c x\} d x \\
& \leq \exp \{-[H(a)-c a]\} \int_{0}^{\infty} \exp (-c x) d x \\
& =\frac{1}{c} \exp \{-[H(a)-c a]\} \quad \forall a \geq A
\end{aligned}
$$

One obtains further with equations (4.7) and (4.6) that

$$
\begin{aligned}
H(a) \int_{a}^{\infty} \exp [-H(x)] d x \leq & \frac{1}{c} H(a) \exp \{-[H(a)-c a]\} \\
\leq & \frac{1}{c}[H(a)-c a] \exp \{-[H(a)-c a]\}+ \\
& a \exp [-(c a-2 c A)] \quad \forall a \geq A
\end{aligned}
$$

By equation (4.6), $\lim _{a \rightarrow \infty} \frac{1}{c}[H(a)-c a] \exp \{-[H(a)-c a]\}=0$, and clearly $\lim _{a \rightarrow \infty} a \exp [-(c a-2 c A)]=0$. Hence $\lim _{a \rightarrow \infty} H(a) \int_{a}^{\infty} \exp [-H(x)] d x=0$, which finishes the proof.

\section{Proof of part (iii)}

By equations (4.3), (1.5), (1.6) and (1.3),

$$
\frac{d}{d a}\left[\frac{d}{d a} \varphi(a)\right]=\frac{d}{d a}\{l(a)[1-H(a)]\}
$$




$$
\begin{aligned}
& =\left[\frac{d}{d a} l(a)\right][1-H(a)]+l(a) \frac{d}{d a}[1-H(a)] \\
& =[-d(a)][1-H(a)]+l(a)[-\mu(a)] \\
& =d(a)[H(a)-1]-l(a) \mu(a) \\
& =d(a)[H(a)-2]
\end{aligned}
$$

So the second derivative of $\varphi$ is strictly negative on $\left[0, a^{*}\right)$ and strictly positive on $\left(a^{*}, \infty\right)$. Thus, $\varphi$ is strictly concave on $\left[0, a^{*}\right]$ and strictly convex on $\left[a^{*}, \infty\right)$.

\subsection{Proof of Theorem 2.2}

Note that $a^{\dagger}<\tilde{a}$ and, by Theorem 2.1(iii), $\varphi$ is strictly concave on $[0, \tilde{a}]$.

By Newton's method, the sequence $\left(x_{n}\right)$, defined by

$$
x_{n+1}=x_{n}-\frac{\varphi\left(x_{n}\right)}{\frac{d}{d a} \varphi\left(x_{n}\right)}, \quad x_{0}=0
$$

strictly increases and rapidly (quadratically) converges to $a^{\dagger}$. In our particular case, by equations (4.2) and (4.1) and Theorem 2.1(i),

$$
\begin{aligned}
x_{n+1} & =x_{n}-\frac{l\left(x_{n}\right) e\left(x_{n}\right) \psi\left(x_{n}\right)}{l\left(x_{n}\right)\left[1-H\left(x_{n}\right)\right]} \\
& =x_{n}-\frac{e\left(x_{n}\right)\left[H\left(x_{n}\right)-1+\frac{e^{\dagger}\left(x_{n}\right)}{e\left(x_{n}\right)}\right]}{1-H\left(x_{n}\right)} \\
& =x_{n}+e\left(x_{n}\right)-\frac{e^{\dagger}\left(x_{n}\right)}{1-H\left(x_{n}\right)}
\end{aligned}
$$

By the Regula Falsi method, the sequence $\left(y_{n}\right)$, defined by

$$
y_{n+1}=y_{n} \cdot \frac{-\varphi(0)}{\varphi\left(y_{n}\right)-\varphi(0)}, \quad y_{0}=\tilde{a}
$$

strictly decreases and (linearly) converges to $a^{\dagger}$. In our particular case, by equations (4.4), (4.2) and (4.1),

$$
\begin{aligned}
y_{n+1} & =y_{n} \cdot \frac{-\left[e^{\dagger}(0)-e(0)\right]}{l\left(y_{n}\right) e\left(y_{n}\right) \psi\left(y_{n}\right)-\left[e^{\dagger}(0)-e(0)\right]} \\
& =y_{n} \cdot \frac{e(0)-e^{\dagger}(0)}{l\left(y_{n}\right) e\left(y_{n}\right)\left[H\left(y_{n}\right)-1+\frac{e^{\dagger}\left(y_{n}\right)}{e\left(y_{n}\right)}\right]+e(0)-e^{\dagger}(0)} \\
& =y_{n} \cdot \frac{e(0)-e^{\dagger}(0)}{l\left(y_{n}\right) e\left(y_{n}\right)\left[H\left(y_{n}\right)-1\right]+l\left(y_{n}\right) e^{\dagger}\left(y_{n}\right)+e(0)-e^{\dagger}(0)}
\end{aligned}
$$




\section{Appendix: Calculating the perturbed life disparity}

Suppose that the cumulative hazard function $H$ gets replaced by $H_{a, s}$, defined in equation (1.7). According to equation (1.6), $\mu$ then gets replaced by $\mu_{a, s}$, where

$$
\mu_{a, s}=\mu-s \cdot \delta_{a}
$$

According to equations (1.4) and (1.6), $l$ then gets replaced by $l_{a, s}$, where

$$
l_{a, s}=l \cdot \exp \left\{s \cdot \mathbb{1}_{[a, \infty)}\right\}
$$

According to equation (1.5), it can be shown that $d$ then gets replaced by $d_{a, s}$, where

$$
d_{a, s}=\left\{1+[\exp (s)-1] \cdot \mathbb{1}_{[a, \infty)}\right\} \cdot d+[1-\exp (s)] l(a) \cdot \delta_{a}
$$

Indeed, for $x<a$, we have $\int_{0}^{x} d_{a, s}(t) d t=\int_{0}^{x} d(t) d t=1-l(x)=1-l_{a, s}(x)$. For $x \geq a$, we have $\int_{0}^{x} d_{a, s}(t) d t=[1-\exp (s)] l(a)+\int_{0}^{a} d(t) d t+\int_{a}^{x} \exp (s) d(t) d t$, where the right hand side simplifies to $[1-\exp (s)] l(a)+[1-l(a)]+\exp (s)[l(a)-$ $l(x)]=1-\exp (s) l(x)=1-l_{a, s}(x)$.

According to equation (1.2), $e$ then gets replaced by $e_{a, s}$, where

$$
e_{a, s}(x)=\frac{1}{l_{a, s}(x)} \int_{x}^{\infty} l_{a, s}(y) d y
$$

By equation (5.1), we obtain

$$
e_{a, s}(x)=\frac{1}{\left(l \cdot \exp \left\{s \cdot \mathbb{1}_{[a, \infty)}\right\}\right)(x)} \int_{x}^{\infty}\left(l \cdot \exp \left\{s \cdot \mathbb{1}_{[a, \infty)}\right\}\right)(y) d y
$$

For $x<a$, we thus have

$$
\begin{aligned}
e_{a, s}(x) & =\frac{1}{l(x)} \int_{x}^{\infty}\left(l \cdot \exp \left\{s \cdot \mathbb{1}_{[a, \infty)}\right\}\right)(y) d y \\
& =\frac{1}{l(x)}\left[\int_{x}^{a} l(y) d y+\int_{a}^{\infty} \exp (s) l(y) d y\right] \\
& =\frac{1}{l(x)}\left[\int_{x}^{\infty} l(y) d y-\int_{a}^{\infty} l(y) d y+\exp (s) \int_{a}^{\infty} l(y) d y\right] \\
& =\frac{1}{l(x)}[l(x) e(x)-l(a) e(a)+\exp (s) l(a) e(a)] \\
& =e(x)+[\exp (s)-1] \frac{l(a)}{l(x)} e(a)
\end{aligned}
$$

Note that if $x<a$ is very close to $a$, then $e_{a, s}(x)$ is approximately $\exp (s) e(a)$. For $x \geq a$, by equation (5.3), we have

$$
e_{a, s}(x)=\frac{1}{\exp (s) l(x)} \int_{x}^{\infty} \exp (s) l(y) d y=\frac{1}{l(x)} \int_{x}^{\infty} l(y) d y=e(x)
$$


Hence, by combining equations (5.4) and (5.5),

$$
e_{a, s}(x)=e(x)+[\exp (s)-1] l(a) e(a) \cdot\left\{\frac{\mathbb{1}_{[0, a)}}{l}\right\}(x)
$$

Finally, according to equation (1.1), $e^{\dagger}$ then gets replaced by $e_{a, s}^{\dagger}$, where

$$
e_{a, s}^{\dagger}=\int_{0}^{\infty} e_{a, s}(x) d_{a, s}(x) d x
$$

By equations (5.2) and (5.6), we obtain

$$
\begin{aligned}
e_{a, s}^{\dagger}= & \int_{0}^{\infty} e(x)\left\{1+[\exp (s)-1] \mathbb{1}_{[a, \infty)}(x)\right\} d(x) d x+ \\
& \int_{0}^{\infty} e(x)[1-\exp (s)] l(a) \delta_{a}(x) d x+ \\
& \int_{0}^{\infty}[\exp (s)-1] l(a) e(a) \frac{\mathbb{1}_{[0, a)}(x)}{l(x)}\left\{1+[\exp (s)-1] \mathbb{1}_{[a, \infty)}(x)\right\} d(x) d x+ \\
& \int_{0}^{\infty}[\exp (s)-1] l(a) e(a) \frac{\mathbb{1}_{[0, a)}(x)}{l(x)}[1-\exp (s)] l(a) \delta_{a}(x) d x \\
= & \left(\int_{0}^{\infty} e(x) d(x) d x+[\exp (s)-1] \int_{a}^{\infty} e(x) d(x) d x\right)+ \\
& e(a)[1-\exp (s)] l(a)+ \\
& {[\exp (s)-1] l(a) e(a) \int_{0}^{a} \frac{d(x)}{l(x)} d x+} \\
& 0
\end{aligned}
$$

Furthermore, by using equations (1.1), (1.3), (1.6) and (1.8), we obtain

$$
\begin{aligned}
e_{a, s}^{\dagger}= & e^{\dagger}+[\exp (s)-1] l(a) e^{\dagger}(a)+[1-\exp (s)] l(a) e(a)+ \\
& {[\exp (s)-1] l(a) e(a) H(a) } \\
= & e^{\dagger}+[\exp (s)-1] l(a) e(a)\left[H(a)-1+\frac{e^{\dagger}(a)}{e(a)}\right]
\end{aligned}
$$

\section{Acknowledgments}

The author greatly thanks James Vaupel, Trifon Missov and Zhen Zhang for helpful comments.

\section{References}

[Dat10] Human Mortality Database. [electronic resource]. University of California, Berkeley (USA) and Max Planck Institute for Demographic Research (Germany). Available at www.mortality.org or www.humanmortality.de (data downloaded on January 7, 2010). 2010 . 
[Key77] Nathan Keyfitz. Applied Mathematical Demography. New York: John Wiley, 1977.

[Mit78] S. Mitra. A short note on the Taeuber paradox. Demography, 15(4):621-623, 1978.

$\left[\mathrm{SAZ}^{+}\right.$09] Vladimir M. Shkolnikov, Evgueni M. Andreev, Zhen Zhang, James Oeppen, and James W. Vaupel. Losses of expected lifetime in the US and other delevoped countries: methods and empirical analyses. MPIDR WORKING PAPER WP 2009-042 DECEMBER 2009, 2009 .

[Vau86] James W. Vaupel. How change in age-specific mortality affects life expectancy. Population Studies, 40(1):147-157, 1986.

[VCR03] James W. Vaupel and Vladimir Canudas-Romo. Decomposing changes in life expectancy: A bouquet of formulas in honor of Nathan Keyfitz's 90th birthday. Demography, 40(2):201-216, 2003.

[ZV08] Zhen Zhang and James W. Vaupel. The Threshold between Compression and Expansion of Mortality. Paper presented at Population Association of America 2008 Annual Meeting, New Orleans, April 17-19, 2008.

[ZV09] Zhen Zhang and James W. Vaupel. The age separating early deaths from late deaths. Demographic Research, 20(29):721-730, 2009. 